\title{
Drone-based Antenna Array for Service Time Minimization in Wireless Networks
}

\author{
Mohammad Mozaffari $^{1}$, Walid Saad ${ }^{1}$, Mehdi Bennis ${ }^{2}$, and Mérouane Debbah ${ }^{3}$ \\ ${ }^{1}$ Wireless@VT, Electrical and Computer Engineering Department, Virginia Tech, VA, USA, Emails: $\{$ mmozaff,walids $\} @$ vt.edu. \\ ${ }^{2}$ CWC - Centre for Wireless Communications, University of Oulu, Finland, Email: bennis@ee.oulu.fi. \\ ${ }^{3}$ Mathematical and Algorithmic Sciences Lab, Huawei France R\&D, Paris, France, and CentraleSupelec, \\ Université Paris-Saclay, Gif-sur-Yvette, France, Email: merouane.debbah@ @uawei.com.
}

\begin{abstract}
In this paper, the effective use of multiple drones as an aerial antenna array that provides wireless service to ground users is investigated. In particular, under the goal of minimizing the service time needed for servicing ground users, a novel framework for deploying a drone-based antenna array system whose elements are single-antenna drones is proposed. To this end, first, the antenna array gain is maximized by optimizing the drone spacing within the array. In this case, using perturbation techniques, the drone spacing optimization problem is addressed by solving successive, perturbed convex optimization problems. In the second step, the optimal locations of the drones around the array's center are derived such that the service time for each ground user is minimized. Simulation results show that the proposed approach can significantly reduce the service time to ground users compared to a single drone that uses the same amount of power as the array. The results also show that the network's spectral efficiency can be improved by $78 \%$ while leveraging the drone antenna array system.
\end{abstract}

\section{INTRODUCTION}

The use of unmanned aerial vehicles (UAVs), popularly known as drones, is growing rapidly across many domains including delivery, communications, and search and rescue in emergency operations [1]-[3]. In wireless networks, drones can be used as flying base stations to provide reliable and costeffective wireless connectivity [1]-[5]. In particular, drones can play a key role in enabling wireless connectivity in various scenarios such as public safety, temporary events (e.g. festivals), and Internet of Things (IoT) scenarios [1]. To effectively use drones for wireless networking applications, one must address a number of challenges that include optimal placement of drones, resource management, and flight time optimization.

Naturally, flying drones have a limited amount of on-board energy which must be used for transmission, mobility, control, and payloads purposes. Consequently, the flight duration of drones is typically short and insufficient for providing a longterm, continuous wireless coverage. Furthermore, due to the limited transmit power of drones, providing long-range, high rate, and low latency communications can be challenging in drone-enabled wireless systems. One promising solution is to utilize multiple drones within an antenna array system in which each element is a single-antenna drone [6]. Clearly, a high gain drone-based antenna array can provide high data

This work was supported in part by the Army Research Office (ARO) under Grant W911NF-17-1-0593, in part by the US NSF under Grant AST1506297, by the ERC Starting Grant MORE (Advanced Mathematical Tools for Complex Network Engineering), and by Academy of Finland (CARMA). rate wireless services to ground users. As a result, the service time, which is defined as the time needed for servicing ground users, will be reduced. From the drones' perspective, a lower service time corresponds to a shorter flight time as well as less energy consumption. From the users' point of view, a lower service time is also preferred as they can be served by the drones within a shorter time, thus experiencing lower latency. Therefore, the service time is a key performance metric in drone-enabled wireless networks as it directly impacts the flight time of drones and quality-of-service (QoS) for users.

Despite the importance of service time in drone communications, current literature still lacks comprehensive studies on the service time analysis. For instance, the work in [7] addressed the flight time optimization problem of drone base stations by deriving the optimal cell association schemes. In [8], the trajectory and mission completion time of a single UAV that serves ground users are optimized. In [6], the authors studied the design of a UAV-based antenna array for directivity maximization. However, the approach presented in [6] is based on a heuristic and a computationally demanding evolutionary algorithm. Moreover, the service time analysis is ignored in [6]. In fact, the previous studies, such as [1]-[8], did not study the service time optimization problem while exploiting a drone-based antenna array system for serving ground users.

The main contribution of this paper is a novel framework for deploying a drone-based antenna array system that delivers wireless service to ground users within a minimum time. In particular, we derive the optimal locations of drones within the array that yield a minimum service time. First, we determine the optimal drone spacing for which the array directivity is maximized. In this case, using the perturbation technique [9], we solve the drone spacing optimization problem by successively solving a number of perturbed convex optimization problems. Next, given the drone spacing, we optimally adjust the locations of the drones according to the position of each ground user. Our results show that the proposed drone antenna array approach can significantly reduce the service time and improve the spectral and energy efficiency of the network.

\section{System Model And Problem Formulation}

Consider a set $\mathcal{L}$ of $L$ single-antenna wireless users located within a given geographical area. In this area, a set $\mathcal{M}$ of $M$ quadrotor drones are used as flying access points to provide downlink wireless service for ground users. The $M$ drones 
will form an antenna array in which each element is a singleantenna drone, as shown in Fig. 1. For tractability, we consider a linear antenna array whose elements are symmetrically excited and located about the origin of the array as done in [10]. The results that we will derive for the linear array case can provide a key guideline for designing more complex two-dimensional (2D) and 3D array configurations. The 3D locations of drone $m \in \mathcal{M}$ and user $i \in \mathcal{L}$ are, respectively, given by $\left(x_{m}, y_{m}, z_{m}\right)$ and $\left(x_{i}^{\mathrm{u}}, y_{i}^{\mathrm{u}}, z_{i}^{\mathrm{u}}\right)$. To avoid collisions between drones, we assume that adjacent drones in the array are separated by at least $D_{\min }$. Let $a_{m}$ and $\beta_{m}$ be the amplitude and phase of the signal (i.e., excitation) at element $m$ in the array. Let $d_{m}=\sqrt{\left(x_{m}-x_{o}\right)^{2}+\left(y_{m}-y_{o}\right)^{2}+\left(z_{m}-z_{o}\right)^{2}}$ be the distance of drone $m$ from the origin of the array whose 3D coordinate is $\left(x_{o}, y_{o}, z_{o}\right)$. The magnitude of the far-field radiation pattern of each element is $w(\theta, \phi)$, where $\theta$ and $\phi$ are the polar and azimuthal angles in the spherical coordinate.

To serve ground users distributed over a geographical area, the drones will dynamically change their positions based on each user's location. Such repositioning is needed for adjusting the distance and beam direction of the antenna array to each ground user. Note that, unlike a classical linear phased array that uses electronic beam steering, the proposed drone-based antenna array relies on the repositioning of drones. This is because in the drone antenna array, precisely adjusting the elements' phase is more challenging than the phased array whose elements are directly connected. Hence, in our model, the drones dynamically adjust their positions in order to steer the beam towards ground users. Clearly, the service time, which is the time needed to serve the ground users, depends on the transmission time and the control time during which the drones must move and stabilize their locations. The transmission time is inversely proportional to the downlink data rate which depends on the signal-to-noise-ratio (SNR) which is, in turn, function of the array's beamforming gain.

The service time is an important metric for both users and drones. A lower service time yields a lower delay and, hence, higher QoS for the users. Also, the service time is directly related to spectral efficiency as it depends on data rate and transmission bandwidth. For drones, a lower service time corresponds to a shorter flight time and less energy consumption. In fact, minimizing the service time improves both energy and spectral efficiency. Therefore, our goal is to minimize the total service time of the ground users by optimally adjusting the drones' locations that can provide a maximum data rate.

For drone-to-ground communications, we consider the classical line-of-sight (LoS) propagation model as also done in [3], [8]. Such channel model is reasonable here as the effect of multipath is significantly mitigated due to the high altitude of drones and using the beamforming technique [8]. The transmission rate from the drone antenna array to ground user $i$ in a far-field region is given by [8]:

$$
R_{i}(\boldsymbol{x}, \boldsymbol{y}, \boldsymbol{z})=B \log _{2}\left(1+\frac{r_{i}^{-\alpha} P_{t} K_{o} G_{i}(\boldsymbol{x}, \boldsymbol{y}, \boldsymbol{z})}{\sigma^{2}}\right),
$$

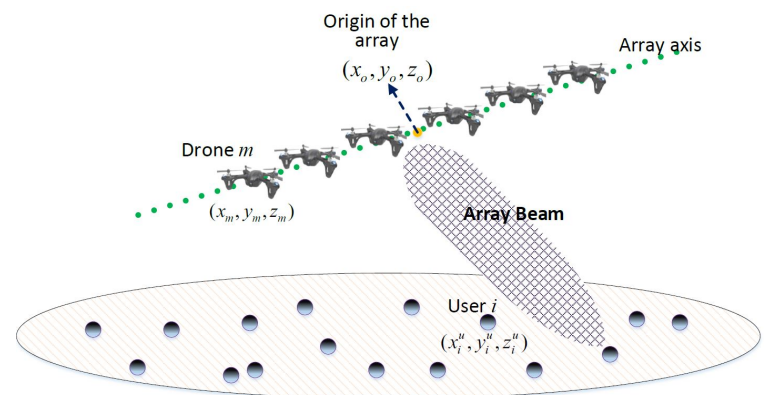

Fig. 1: Drone-based antenna array.

where $\boldsymbol{x}=\left[x_{m}\right]_{M \times 1}, \boldsymbol{y}=\left[y_{m}\right]_{M \times 1}, \boldsymbol{z}=\left[z_{m}\right]_{M \times 1}, m \in \mathcal{M}$ are vectors of $x, y$, and $z$ coordinates of the drones. $B$ is the transmission bandwidth, $r_{i}$ is the distance between the origin of the array and user $i, P_{t}$ is the total transmit power of the array, $\sigma^{2}$ is the noise power, and $K_{o}$ is the constant path loss coefficient. $G_{i}\left(x_{m}, y_{m}, z_{m}\right)$ is the gain of the antenna array towards the location of user $i$, given by [11]:

$$
G_{i}(\boldsymbol{x}, \boldsymbol{y}, \boldsymbol{z})=\frac{4 \pi\left|F\left(\theta_{i}, \phi_{i}\right)\right|^{2} w\left(\theta_{i}, \phi_{i}\right)^{2}}{\int_{0}^{2 \pi} \int_{0}^{\pi}|F(\theta, \phi)|^{2} w(\theta, \phi)^{2} \sin \theta \mathrm{d} \theta \mathrm{d} \phi} \eta
$$

where $0 \leq \eta \leq 1$ is the antenna array efficiency which is multiplied by directivity to compute the antenna gain. In fact, the antenna gain is equal to the antenna directivity multiplied by $\eta$. In (2), $F(\theta, \phi)$ is the array factor given by [11]:

$F(\theta, \phi)=\sum_{m=1}^{M} a_{m} e^{j\left[k\left(x_{m} \sin \theta \cos \phi+y_{m} \sin \theta \sin \phi+z_{m} \cos \theta\right)+\beta_{m}\right]}$,

where $k=2 \pi / \lambda$ is the phase constant, and $\lambda$ is the wavelength. The overall radiation pattern of the antenna array is equal to $F(\theta, \phi) w\left(\theta_{i}, \phi_{i}\right)$ which follows from the pattern multiplication rule [11]. Now, the total service time $T_{\text {tot }}$ is:

$$
T_{\text {tot }}=\sum_{i=1}^{L} \frac{q_{i}}{R_{i}(\boldsymbol{x}, \boldsymbol{y}, \boldsymbol{z})}+T^{\mathrm{crl}},
$$

where $q_{i}$ is the load of user $i$ representing the number of bits that must be transmitted to user $i$. $T^{\mathrm{crl}}$ is the total control time during which the drones adjust their locations according to the users' locations. Here, we assume that the total control time is given based on the locations and the number of users.

Our goal is to minimize the total service time of drones by minimizing the transmission time. In this case, we must find the optimal locations of the drones, with respect to the center of the array, that yield a minimum drone transmission time:

$$
\begin{aligned}
& \min _{\boldsymbol{x}, \boldsymbol{y}, \boldsymbol{z}} \sum_{i=1}^{L} \frac{q_{i}}{R_{i}(\boldsymbol{x}, \boldsymbol{y}, \boldsymbol{z})}, \\
\text { st. } & d_{m+1}-d_{m} \geq D_{\min }, \forall m \in \mathcal{M} \backslash\{M\},
\end{aligned}
$$

where (6) is a constraint on the minimum separation distance of two adjacent drones needed to avoid collision.

Solving (5) is challenging as it is highly nonlinear due to (2). Moreover, as we can see from (3), the array factor is a complex function of the array element's positions. Clearly, given (1), (2), and (4), to minimize the transmission time, we 
need to maximize the array gain towards each ground user. In other words, we determine the optimal positions of drones based on the location of each user such that the transmission time to the user is minimized. As a result, given $L$ ground users, we will have $L$ sets of drones' locations. In the next section, we will optimize the position of drones to maximize the array directivity towards any given ground user.

\section{Optimal DRone Positions}

Here, we maximize the directivity of the drone-based antenna array towards a given ground user by optimally adjusting the drones' locations. Without loss of generality, we consider an even number of drones, but the analysis still holds for an odd number. Now, the array factor for $M$ drones located on the $x$-axis of the Cartesian coordinate will be:

$$
\begin{aligned}
& F(\theta, \phi)=\sum_{m=1}^{M} a_{m} e^{j\left[k x_{m} \sin \theta \cos \phi+\beta_{m}\right]} \\
& \stackrel{(a)}{=} \sum_{n=1}^{M / 2} a_{n}\left(e^{j\left[k d_{n} \sin \theta \cos \phi+\beta_{n}\right]}+e^{-j\left[k d_{n} \sin \theta \cos \phi+\beta_{n}\right]}\right) \\
& \stackrel{(b)}{=} 2 \sum_{n=1}^{N} a_{n} \cos \left(k d_{n} \sin \theta \cos \phi+\beta_{n}\right),
\end{aligned}
$$

where $N=M / 2$, and $d_{n}$ is the distance of element $n \in$ $\mathcal{N}=\{1,2, \ldots, N\}$ from the center of the array (origin). Also, (a) follows from the fact that the array is symmetric with respect to the origin, and $(b)$ is based on the Euler's rule. We can maximize the directivity of the array by optimizing $d_{n}$, $\forall n \in \mathcal{N}:$

$$
\max _{d_{n}, \forall n \in \mathcal{N}} \frac{4 \pi\left|F\left(\theta_{\max }, \phi_{\max }\right)\right|^{2} w\left(\theta_{\max }, \phi_{\max }\right)^{2}}{\int_{0}^{2 \pi} \int_{0}^{\pi}|F(\theta, \phi)|^{2} w(\theta, \phi)^{2} \sin \theta \mathrm{d} \theta \mathrm{d} \phi},
$$

where $\left(\theta_{\max }, \phi_{\max }\right)$ are the polar and azimuthal angles at which the total antenna pattern $F(\theta, \phi) w(\theta, \phi)$ has a maximum value. Solving (8) is challenging due to the non-linearity and complex expression of the objective function. In fact, this problem is non-convex and cannot be exactly solved using classical convex optimization methods. Next, we provide an analytical solution to (8) by exploiting the perturbation technique [10]. In general, perturbation theory aims at finding the solution of a complex problem, by starting from the exact solution of a simplified version of the original problem [9]. This technique is thus useful when dealing with non-linear optimization problems such as (8).

\section{A. Perturbation Technique for Drone Spacing Optimization}

To optimize the distance between drones, we first consider an initial value for the distance of each drone from the origin. Then, we find the optimal perturbation value that must be added to this initial value. Let $d_{n}^{0}$ be initial distance of drone $n$ from the origin, then, the perturbed distance will be:

$$
d_{n}=d_{n}^{0}+e_{n},
$$

where $e_{n}<<\lambda$, with $\lambda$ the wavelength, is the perturbation value. Given (9), the array factor can be approximated by:

$$
\begin{aligned}
& F(\theta, \phi)=2 \sum_{n=1}^{N} a_{n} \cos \left(k\left(d_{n}^{0}+e_{n}\right) \sin \theta \cos \phi+\beta_{n}\right) \\
& =2 \sum_{n=1}^{N} a_{n} \cos \left[\left(k d_{n}^{0} \sin \theta \cos \phi+\beta_{n}\right)+k e_{n} \sin \theta \cos \phi\right] \\
& \stackrel{(a)}{\approx} \sum_{n=1}^{N} 2 a_{n} \cos \left(k d_{n}^{0} \sin \theta \cos \phi+\beta_{n}\right) \\
& \quad-\sum_{n=1}^{N} 2 a_{n} k e_{n} \sin \theta \cos \phi \sin \left(k d_{n}^{0} \sin \theta \cos \phi+\beta_{n}\right),
\end{aligned}
$$

where in $(a)$ we used the trigonometric properties, and the fact that $\sin (x) \approx x$ for small values of $x$.

Clearly, given $e_{n}<<\lambda$, the numerator of (8) can be computed based on the values of $d_{n}^{0}, \forall n \in \mathcal{N}$. Hence, given $d_{n}^{0}$, our optimization problem in (8) can be written as:

$$
\begin{array}{cl} 
& \min _{e} \int_{0}^{2 \pi} \int_{0}^{\pi} F(\theta, \phi)^{2} w(\theta, \phi)^{2} \sin \theta \mathrm{d} \theta \mathrm{d} \phi, \\
\text { s.t. } & d_{n+1}^{0}+e_{n+1}-d_{n}^{0}-e_{n} \geq D_{\min }, \forall n \in \mathcal{N} \backslash\{N\},
\end{array}
$$

where $e$ is the perturbation vector having elements $e_{n}, n \in \mathcal{N}$.

For brevity, we define the following functions:

$$
\begin{aligned}
& F^{0}(\theta, \phi)=\sum_{n=1}^{N} a_{n} \cos \left(k d_{n}^{0} \sin \theta \cos \phi+\beta_{n}\right), \\
& I_{\text {int }}(x)=\int_{0}^{2 \pi} \int_{0}^{\pi} x \sin \theta \mathrm{d} \theta \mathrm{d} \phi .
\end{aligned}
$$

Theorem 1. The optimization problem in (11) is convex, and the optimal perturbation vector is the solution of the following system of equations:

$$
\left\{\begin{array}{l}
\boldsymbol{e}=\boldsymbol{G}^{-1}\left[\boldsymbol{q}+\boldsymbol{\mu}_{\mathcal{L}}\right], \\
\mu_{n}\left(e_{n}-e_{n+1}+D_{\min }+d_{n}^{0}-d_{n+1}^{0}\right)=0, \forall n \in \mathcal{N} \backslash\{N\}, \\
\mu_{n} \geq 0, \forall n \in \mathcal{N} \backslash\{N\},
\end{array}\right.
$$

where $\boldsymbol{G}=\left[g_{m, n}\right]_{N \times N}$ is an $N \times N$ matrix with:

$$
\begin{aligned}
& g_{m, n}=I_{\text {int }}\left(a_{m} a_{n}(k \sin \theta \cos \phi w(\theta, \phi))^{2}\right. \\
& \left.\times \sin \left(k d_{n}^{0} \sin \theta \cos \phi+\beta_{n}\right) \sin \left(k d_{m}^{0} \sin \theta \cos \phi+\beta_{m}\right)\right),
\end{aligned}
$$

and $\boldsymbol{q}=\left[q_{n}\right]_{N \times 1}$ whose elements are given by:

$$
\begin{aligned}
q_{n}=I_{\text {int }}( & a_{n} k \sin \theta \cos \phi w(\theta, \phi) F^{0}(\theta, \phi) \\
& \left.\times \sin \left(k d_{n}^{0} \sin \theta \cos \phi+\beta_{n}\right)\right) .
\end{aligned}
$$

$\boldsymbol{\mu}_{\mathcal{L}}$ is a vector of Lagrangian multipliers, whose element $n$ is: $\boldsymbol{\mu}_{\mathcal{L}}(n)=\mu_{n+1}-\mu_{n}$ where $\mu_{n}$ is a Lagrangian multiplier associated with constraint $n$.

Proof: First, we find $F^{2}(\theta, \phi)$ by using (10):

$$
F^{2}(\theta, \phi)=\left[2 F^{0}(\theta, \phi)\right]^{2}+
$$




$$
\begin{array}{r}
{\left[2 \sum_{n=1}^{N} a_{n} k e_{n} \sin \theta \cos \phi \sin \left(k d_{n}^{0} \sin \theta \cos \phi+\beta_{n}\right)\right]^{2}} \\
-8 F^{0}(\theta, \phi) \sum_{n=1}^{N} a_{n} k e_{n} \sin \theta \cos \phi \sin \left(k d_{n}^{0} \sin \theta \cos \phi+\beta_{n}\right) .
\end{array}
$$

Subsequently, our objective function in (11) can be written as:

$$
\begin{aligned}
& I_{\text {int }}\left(F^{2}(\theta, \phi) w^{2}(\theta, \phi)\right)= \\
& \quad 4\left[\boldsymbol{e}^{T} \boldsymbol{G e}-2 \boldsymbol{e}^{T} \boldsymbol{q}+I_{\mathrm{int}}\left(F_{0}^{2}(\theta, \phi) w^{2}(\theta, \phi)\right)\right],
\end{aligned}
$$

where $\boldsymbol{G}$ and $\boldsymbol{q}$ are given in (16) and (17). Clearly, (18) is a quadratic function of $e$. Therefore, (18) is convex if and only if $\boldsymbol{G}$ is a positive semi-definite matrix. Given (16), we have:

$$
\boldsymbol{y}^{T} \boldsymbol{G} y=\sum_{n=1}^{N} y_{n} \sum_{m=1}^{N} y_{m} g_{m, n} \text {. }
$$

Now, in (16), let us define

$z_{n}=a_{n} k \sin \theta \cos \phi w(\theta, \phi) \sin \left(k d_{n}^{0} \sin \theta \cos \phi+\beta_{n}\right)$,

then, using (19), we have:

$$
\boldsymbol{y}^{T} \boldsymbol{G} \boldsymbol{y}=I_{\mathrm{int}}\left(\left[\sum_{n=1}^{N} z_{n} y_{n}\right]^{2}\right) \text {. }
$$

In (14), we can see that $I_{\text {int }}(x) \geq 0$ for $x \geq 0$. Hence, from (21), we can conclude that $\boldsymbol{y}^{T} \boldsymbol{G} \boldsymbol{y} \geq 0$. Therefore, $\boldsymbol{G}$ is positive semi-definite and the objective function in (11) is convex. Moreover, the constraints in (12) are affine functions which are convex. Hence, this optimization problem is convex.

Now, we find the optimal perturbation vector $e$ by using Karush-Kuhn-Tucker (KKT) conditions. The Lagrangian function will be:

$$
\begin{aligned}
\mathcal{L}= & \boldsymbol{e}^{T} \boldsymbol{G e}-2 \boldsymbol{e}^{T} \boldsymbol{q}+I_{\text {int }}\left(F_{0}^{2}(\theta, \phi) w^{2}(\theta, \phi)\right) \\
& +\sum_{n=1}^{N-1} \mu_{n}\left(e_{n}-e_{n+1}+D_{\min }+d_{n}^{0}-d_{n+1}^{0}\right),
\end{aligned}
$$

where $\mu_{n} \geq 0, n=1, \ldots, N-1$ are the Lagrange multipliers.

The necessary and sufficient (due to the convexity of the problem) KKT conditions for finding the optimal perturbation vector $e$ are given by:

$$
\nabla_{\boldsymbol{e}}[\mathcal{L}]=0,
$$

which leads to $\boldsymbol{e}=\boldsymbol{G}^{-1}\left[\boldsymbol{q}+\boldsymbol{\mu}_{\mathcal{L}}\right]$, with $\boldsymbol{\mu}_{\mathcal{L}}$ being a $(N-1) \times 1$ vector whose element $n$ is $\boldsymbol{\mu}_{\mathcal{L}}(n)=\mu_{n+1}-\mu_{n}$.

Based on the complementary slackness conditions, we have: $\left\{\begin{array}{l}\mu_{n}\left(e_{n}-e_{n+1}+D_{\min }+d_{n}^{0}-d_{n+1}^{0}\right)=0, \forall n \in \mathcal{N} \backslash\{N\}, \\ \mu_{n} \geq 0, \forall n \in \mathcal{N} \backslash\{N\} .\end{array}\right.$

Finally, the optimal perturbation vector, $e^{*}$, can be determined by solving (23) and (24). This proves the theorem.

Using Theorem 1, we can update the distance of each drone from the origin:

$$
d^{1}=d^{0}+e^{*}
$$

where $\boldsymbol{d}^{1}=\left[d_{n}^{1}\right]_{N \times 1}$, and $\boldsymbol{d}^{0}=\left[d_{n}^{0}\right]_{N \times 1}, n \in \mathcal{N}$.

Clearly, $\boldsymbol{d}^{1}$ leads to a better solution than $\boldsymbol{d}^{0}=\left[d_{n}\right]_{N \times 1}$. In fact, we can proceed and further improve the solution to (11) by updating $\boldsymbol{d}^{1}$. In particular, at update step $r \in \mathbb{N}$, we find $\boldsymbol{d}^{(r)}$ as follows:

$$
\boldsymbol{d}^{(r)}=\boldsymbol{d}^{(r-1)}+\boldsymbol{e}^{*(r)}
$$

where $e^{*(r)}$ is the optimal perturbation vector at step $r$ which is obtained based on $\boldsymbol{d}^{(r-1)}$.

Note that, at each step, the objective function in (11) decreases. Since the objective function is monotonically decreasing and bounded from below, the solution converges after several updates. We note that due to the approximation used in (10), the solution may not be a global optimal. Nevertheless, as we can see from Theorem 1, it is analytically tractable and, hence, it has a low computational complexity. Here, we use $\boldsymbol{d}^{*}$ to represent the vector of nearly-optimal distances of drones from the original of the array. Next, we use $\boldsymbol{d}^{*}$ to determine the optimal 3D locations of the drones that result in a maximum array directivity towards a given ground user.

\section{B. Optimal Locations of Drones}

Here, following from Subsection III-A, we derive the optimal 3D positions of drones that yields a maximum directivity of the drone-based antenna array. Let $\left(x_{i}^{\mathrm{u}}, y_{i}^{\mathrm{u}}, z_{i}^{\mathrm{u}}\right)$ and $\left(x_{o}, y_{o}, z_{o}\right)$ be, respectively, the $3 \mathrm{D}$ locations of user $i \in \mathcal{L}$ and the origin of the antenna array.

Without loss of generality, we translate the origin of our coordinate system to the origin of the antenna array. In other words, we assume that the arrays' center is the origin of our translated coordinate system. In this case, the 3D location of user $i$ will be $\left(x_{i}^{\mathrm{u}}-x_{o}, y_{i}^{\mathrm{u}}-y_{o}, z_{i}^{\mathrm{u}}-z_{o}\right)$. Subsequently, the polar and azimuthal angles of user $i$ in the spherical coordinate (with an origin of antenna array) are given by:

$$
\begin{aligned}
& \theta_{i}=\cos ^{-1}\left[\frac{z_{i}^{u}-z_{o}}{\sqrt{\left(x_{i}^{u}-x_{o}\right)^{2}+\left(y_{i}^{u}-y_{o}\right)^{2}+\left(z_{i}^{u}-z_{o}\right)^{2}}}\right], \\
& \phi_{i}=\sin ^{-1}\left[\frac{y_{i}^{u}-y_{o}}{\sqrt{\left(x_{i}^{u}-x_{o}\right)^{2}+\left(y_{i}^{u}-y_{o}\right)^{2}}}\right] .
\end{aligned}
$$

Now, the optimal locations of the drones in the antenna array is given as follows.

Theorem 2. The optimal locations of the drones for maximizing the directivity of the drone-based antenna array towards a given ground user will be:

$$
\begin{aligned}
& \left(x_{m}^{*}, y_{m}^{*}, z_{m}^{*}\right)^{T}= \\
& \left\{\begin{array}{l}
\boldsymbol{R}_{\mathrm{rot}}\left(d_{m}^{*} \sin \alpha_{o} \cos \gamma_{o}, d_{m}^{*} \sin \alpha_{o} \sin \beta_{o}, d_{m}^{*} \cos \alpha_{o}\right)^{T}, m \leq M / 2, \\
-\boldsymbol{R}_{\mathrm{rot}}\left(d_{m}^{*} \sin \alpha_{o} \cos \gamma_{o}, d_{m}^{*} \sin \alpha_{o} \sin \gamma_{o}, d_{m}^{*} \cos \alpha_{o}\right)^{T}, m>M / 2,
\end{array}\right.
\end{aligned}
$$

where $\alpha_{o}$ and $\gamma_{o}$ are the initial polar and azimuthal angles of drone $m \leq M / 2$ with respect to the array's center. $\boldsymbol{R}_{\text {rot }}$ is the rotation matrix for updating drones' positions, given by:

$$
\begin{aligned}
& \boldsymbol{R}_{\mathrm{rot}}= \\
& \left(\begin{array}{ccc}
a_{x}^{2}(1-\delta)+\delta & a_{x} a_{y}(1-\delta)-\lambda a_{z} & a_{x} a_{z}(1-\delta)+\lambda a_{y} \\
a_{x} a_{y}(1-\delta)+\lambda a_{z} & a_{y}^{2}(1-\delta)+\delta & a_{y} a_{z}(1-\delta)-\lambda a_{x} \\
a_{x} a_{z}(1-\delta)-\lambda a_{y} & a_{y} a_{z}(1-\delta)+\lambda a_{x} & a_{z}^{2}(1-\delta)+\delta
\end{array}\right),
\end{aligned}
$$


where $\delta=\left\|\boldsymbol{q}_{\boldsymbol{i}} \cdot \boldsymbol{q}_{\max }\right\|, \lambda=\sqrt{1-\delta^{2}}, \boldsymbol{q}_{\boldsymbol{i}}=\left(\begin{array}{c}\sin \theta_{i} \cos \phi_{i} \\ \sin \theta_{i} \sin \phi_{i} \\ \cos \theta_{i}\end{array}\right)$, $\boldsymbol{q}_{\max }=\left(\begin{array}{c}\sin \theta_{\max } \cos \phi_{\max } \\ \sin \theta_{\max } \sin \phi_{\max } \\ \cos \theta_{\max }\end{array}\right)$. Moreover, $a_{x}, a_{y}$, and $a_{z}$ are the elements of vector $\boldsymbol{a}=\left(a_{x}, a_{y}, a_{z}\right)^{T}=\boldsymbol{q}_{\boldsymbol{i}} \times \boldsymbol{q}_{\max }$.

Proof: In Subsection III-A, we have derived the optimal distance of drones from the origin that leads to a maximum array directivity. First, we consider an initial (or arbitrary) orientation, as shown in Fig. 2. Let $d_{m}^{*}$ be the optimal distance of drone $m \leq M / 2$ from the array's center, $\alpha_{o}$ and $\gamma_{o}$ be the initial polar and azimuthal angles of the drone. Based on the considered drones' locations, let $\left(\theta_{\max }, \phi_{\max }\right)=\operatorname{argmax}[F(\theta, \phi) w(\theta, \phi)]$ be a direction at which the directivity of the array is maximized. Our goal is to achieve the maximum directivity at a given direction $\left(\theta_{i}, \phi_{i}\right)$ corresponding to user $i$. Therefore, we need to change the locations of the drones such that $\theta_{i}=\theta_{\max }$, and $\phi_{i}=\phi_{\max }$. To this end, we align the unit vector $\left(1, \theta_{\max }, \phi_{\max }\right)$ with $\left(1, \theta_{i}, \phi_{i}\right)$ in the spherical coordinate and, then, we update the drones' positions accordingly. In the Cartesian coordinate system, we need to rotate vector $\boldsymbol{q}_{\max }=$ $\left(\sin \theta_{\max } \cos \phi_{\max }, \sin \theta_{\max } \sin \phi_{\max }, \cos \theta_{\max }\right)^{T}$ such that it becomes aligned with $\boldsymbol{q}_{i}=\left(\sin \theta_{i} \cos \phi_{i}, \sin \theta_{i} \sin \phi_{i}, \cos \theta_{i}\right)^{T}$.

The rotation matrix for rotating a vector $\boldsymbol{u}$ about another vector $\boldsymbol{a}=\left(a_{x}, a_{y}, a_{z}\right)^{T}$, with a $\omega$ rotation angle, is [12]:

$$
\boldsymbol{R}_{\mathrm{rot}}=\left(\begin{array}{lll}
\boldsymbol{R}_{\mathrm{rot}, 1} & \boldsymbol{R}_{\mathrm{rot}, 2} & \boldsymbol{R}_{\mathrm{rot}, 3}
\end{array}\right),
$$

$$
\text { where } \quad \boldsymbol{R}_{\mathrm{rot}, 1}=\left(\begin{array}{c}
a_{x}^{2}(1-\cos \omega)+\cos \omega \\
a_{x} a_{y}(1-\cos \omega)+a_{z} \sin \omega \\
a_{x} a_{z}(1-\cos \omega)-a_{y} \sin \omega
\end{array}\right) \text {, }
$$$$
\boldsymbol{R}_{\mathrm{rot}, 2}=\left(\begin{array}{c}
a_{x} a_{y}(1-\cos \omega)-a_{z} \sin \omega \\
a_{y}^{2}(1-\cos \omega)+\cos \omega \\
a_{y} a_{z}(1-\cos \omega)+a_{x} \sin \omega
\end{array}\right), \quad \text { and } \quad \boldsymbol{R}_{\mathrm{rot}, 3}=
$$$$
\left(\begin{array}{c}
a_{x} a_{z}(1-\cos \omega)+a_{y} \sin \omega \\
a_{y} a_{z}(1-\cos \omega)-a_{x} \sin \omega \\
a_{z}^{2}(1-\cos \omega)+\cos \omega
\end{array}\right) .
$$

In our problem, the rotation between $\boldsymbol{q}_{\max }$ and $\boldsymbol{q}_{\boldsymbol{i}}$ can be done about the normal vector of these vectors, with the rotation angle being the angle between $\boldsymbol{q}_{\max }$ and $\boldsymbol{q}_{\boldsymbol{i}}$. Hence, based on the dot-product and cross-product of vectors, we use $\boldsymbol{a}=\boldsymbol{q}_{\boldsymbol{i}} \times \boldsymbol{q}_{\max }$, and $\omega=\cos ^{-1}\left(\boldsymbol{q}_{\boldsymbol{i}} \cdot \boldsymbol{q}_{\max }\right)$ to find the rotation matrix in (31). Now, we update the locations of drones using the rotation matrix. Clearly, for $m \leq M / 2$, the initial location of drone $m$ in the Cartesian coordinate is $\left(d_{m}^{*} \sin \alpha_{o} \cos \gamma_{o}, d_{m}^{*} \sin \alpha_{o} \sin \beta_{o}, d_{m}^{*} \cos \alpha_{o}\right)^{T}$. As a result, the optimal locations of drones for serving user $i$ is given by:

$\left(x_{m}^{*}, y_{m}^{*}, z_{m}^{*}\right)^{T}=$

$\boldsymbol{R}_{\text {rot }}\left(d_{m}^{*} \sin \alpha_{o} \cos \gamma_{o}, d_{m}^{*} \sin \alpha_{o} \sin \beta_{o}, d_{m}^{*} \cos \alpha_{o}\right)^{T}, m \leq M / 2$.

Finally, due to the symmetric configuration of the antenna array about the origin, the optimal locations of drones $m$ when $m>M / 2$ are as follows:

$\left(x_{m}^{*}, y_{m}^{*}, z_{m}^{*}\right)^{T}=$

$-\boldsymbol{R}_{\text {rot }}\left(d_{m}^{*} \sin \alpha_{o} \cos \gamma_{o}, d_{m}^{*} \sin \alpha_{o} \sin \beta_{o}, d_{m}^{*} \cos \alpha_{o}\right)^{T}, m \leq M / 2$.

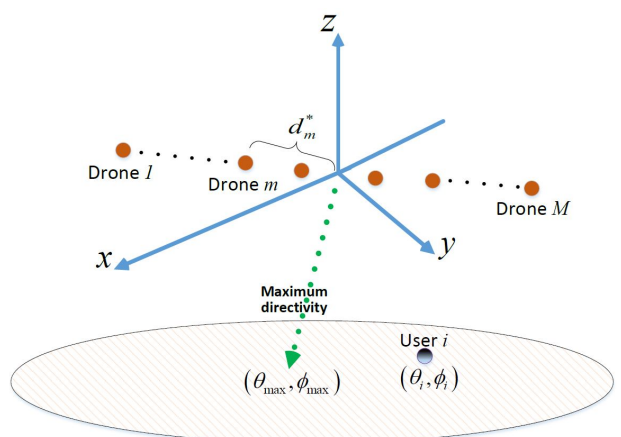

Fig. 2: Illustrative figure for Theorem 2.

Algorithm 1 Optimizing drones' locations for maximum array gain towards user $i$.

1: Inputs: Locations of user $i,\left(x_{i}^{\mathrm{u}}, y_{i}^{\mathrm{u}}, z_{i}^{\mathrm{u}}\right)$, and origin of array, $\left(x_{o}, y_{o}, z_{o}\right)$.

2: Outputs: Optimal drones' positions, $\left(x_{m, i}^{*}, y_{m, i}^{*}, z_{m, i}^{*}\right), \forall m \in$ $\mathcal{M}$.

3: Set initial values for distance between drones, $\boldsymbol{d}$.

4: Find $e^{*}$ by using (15)-(17).

5: Update $\boldsymbol{d}$ based on (25).

6: Repeat steps (4) and (5) to find the optimal spacing vector $\boldsymbol{d}^{*}$.

7: Use (27)-(31) to determine $\left(x_{m}^{*}, y_{m}^{*}, z_{m}^{*}\right), \forall m \in \mathcal{M}$.

Using Theorem 2, we can find the optimal locations of the drones such that the directivity of the drone-based antenna array is maximized towards any given ground user. This, in turn, allows maximizing the data rate and, hence, the transmission time for serving the user is minimized. In Algorithm 1 , we have summarized the key steps needed for optimizing the locations of drones with respect to the center of the array.

\section{Simulation Results And AnAlysis}

For our simulations, we consider 100 ground users which are uniformly distributed within a square area of size $1 \mathrm{~km} \times$ $1 \mathrm{~km}$. Unless stated otherwise, the number of drones ${ }^{1}$ that form a linear array is assumed to be 10 . The main simulation parameters are given in Table I. We compare the performance of our drone-based antenna array system with a single drone that uses the same total transmit power as the array. All statistical results are averaged over a large number of independent runs.

Fig. 3 shows the total service time for the drone antenna array and the single drone case. For a fair comparison, the transmit power of the single drone case is equal to the sum of transmit power of the array's drones. Also, we do not consider any control time for the single drone. Fig. 3 shows that, for a given bandwidth, our proposed drone antenna array outperforms the single drone case in terms of service time. This is because, in the proposed approach, the drones' locations are adjusted such that the array antenna gain towards each user is maximized, hence reducing the transmission time. Fig. 3 also shows the tradeoff between bandwidth and service time. Clearly, the service time decreases by using more bandwidth which effectively provides a higher data rate. Fig. 3 shows that

${ }^{1}$ In our simulations, each drone has an omni-directional antenna. 


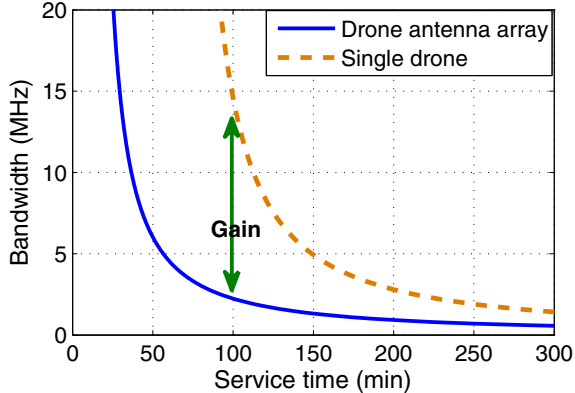

Fig. 3: Service time vs. bandwidth.

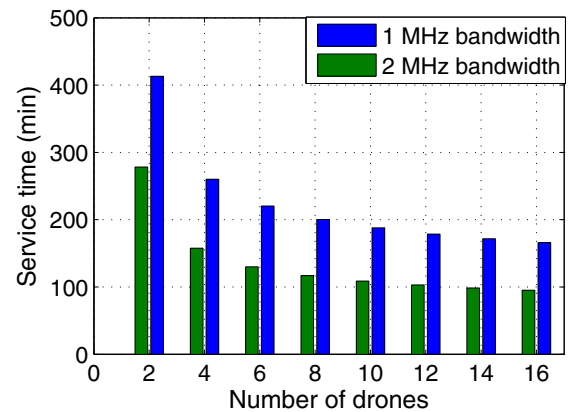

Fig. 4: Service time vs. number of drones.

the drone antenna array improves spectral efficiency compared to the single drone case. For instance, to achieve 100 minutes of service time, the drone antenna array will require $78 \%$ less bandwidth than the single drone.

In Fig. 4, we show the impact of the number of drones on the service time. By increasing the number of drones, the array gain will increase which yields a lower service time. Fig. 4 shows that the service time decreases by $57 \%$ for $1 \mathrm{MHz}$ bandwidth when the number of drones increases from 2 to 10 . In this case, to meet a given service time requirement, one can reduce the bandwidth by deploying more drones in the array. Fig. 4 shows that a case with 4 drones and $1 \mathrm{MHz}$ bandwidth outperforms the one with 2 drones and $2 \mathrm{MHz}$ bandwidth. Therefore, the spectral efficiency can be improved at the cost of using more drones.

Fig. 5 shows the impact of the control time on the total service time while employing the drone-based antenna array. Clearly, in the single drone case with an omni-directional antenna, the control time is negligible. In the drone antenna array, however, the service time increases while increasing the control time. In fact, the drone antenna array outperforms the single drone case if the service time reduction due to the array gain is less than the additional control time. For instance, for $1 \mathrm{~Gb}$ load, as long as the total control time is less than 180 min, the drone antenna array yields a lower service time than the single drone. This figure also shows that by increasing the load, the total service time increases.

\section{CONCLUSION}

In this paper, we have proposed a novel framework for employing a drone-enabled antenna array system that can provide high rate (i.e., low service time) wireless services to ground users. In particular, we have optimized the positions of drones (as the array elements) within the antenna array

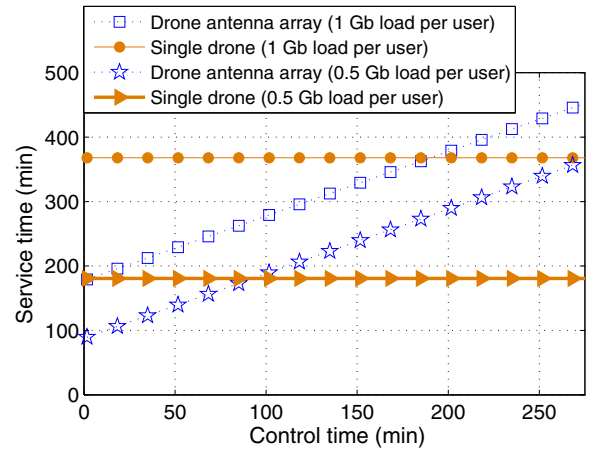

Fig. 5: Service time as a function of the control time.

Table I: Main simulation parameters.

\begin{tabular}{|c|c|c|}
\hline Parameter & Description & Value \\
\hline \hline$f_{c}$ & Carrier frequency & $700 \mathrm{MHz}$ \\
\hline$P_{i}$ & Drone transmit power & $0.1 \mathrm{~W}$ \\
\hline$N_{o}$ & Total noise power spectral density & $-160 \mathrm{dBm} / \mathrm{Hz}$ \\
\hline$N$ & Number of ground users & 100 \\
\hline$B$ & Bandwidth & $1 \mathrm{MHz}$ \\
\hline$T^{\text {crl }}$ & Total control time & $500 \mathrm{~s}$ \\
\hline$\left(x_{o}, y_{o}, z_{o}\right)$ & Array's center coordinate & $(0,0,100)$ in meters \\
\hline$q_{i}$ & Load per user & $1 \mathrm{~Gb}$ \\
\hline$\alpha$ & Pathloss exponent & 3 \\
\hline$\beta_{m}-\beta_{m-1}$ & Phase excitation difference of consequence antennas & $\frac{\pi}{5(M-1)}$ \\
\hline
\end{tabular}

such that the service time for each user is minimized. Our results have shown that the proposed drone antenna array with the optimal configuration yields a significant improvement in terms of the service time, spectral and energy efficiency.

\section{REFERENCES}

[1] M. Mozaffari, W. Saad, M. Bennis, and M. Debbah, "Unmanned aerial vehicle with underlaid device-to-device communications: Performance and tradeoffs," IEEE Transactions on Wireless Communications, vol. 15, no. 6, pp. 3949-3963, June 2016.

[2] M. Alzenad, A. El-Keyi, and H. Yanikomeroglu, "3D placement of an unmanned aerial vehicle base station for maximum coverage of users with different QoS requirements," IEEE Wireless Communications Letters, Early access, 2017.

[3] Q. Wu, Y. Zeng, and R. Zhang, "Joint trajectory and communication design for UAV-enabled multiple access," IEEE Transactions on Wireless Communications, Early access, 2017.

[4] M. M. Azari, F. Rosas, K. C. Chen, and S. Pollin, "Joint sum-rate and power gain analysis of an aerial base station," in Proc. of IEEE Global Communications Conference (GLOBECOM) Workshops, Washington, DC, Dec. 2016.

[5] M. Mozaffari, W. Saad, M. Bennis, and M. Debbah, "Efficient deployment of multiple unmanned aerial vehicles for optimal wireless coverage," IEEE Communications Letters, vol. 20, no. 8, pp. 1647-1650, Aug. 2016.

[6] J. Garza, M. A. Panduro, A. Reyna, G. Romero, and C. d. Rio, "Design of UAVs-based 3D antenna arrays for a maximum performance in terms of directivity and SLL," International Journal of Antennas and Propagation, vol. 2016, Aug. 2016.

[7] M. Mozaffari, W. Saad, M. Bennis, and M. Debbah, "Wireless communication using unmanned aerial vehicles (UAVs): Optimal transport theory for hover time optimization," IEEE Transactions on Wireless Communications, vol. 16, no. 12, pp. 8052-8066, Dec. 2017.

[8] Y. Zeng, X. Xu, and R. Zhang, "Trajectory optimization for completion time minimization in UAV-enabled multicasting," available online: arxiv.org/abs/1708.06478, 2017.

[9] J. F. Bonnans and A. Shapiro, "Optimization problems with perturbations: A guided tour," SIAM review, vol. 40, no. 2, pp. 228-264, 1998.

[10] D. K. Cheng, "Optimization techniques for antenna arrays," Proceedings of the IEEE, vol. 59, no. 12, pp. 1664-1674, Dec. 1971.

[11] W. L. Stutzman and G. A. Thiele, Antenna theory and design. John Wiley \& Sons, 2012.

[12] T. Bajd, M. Mihelj, and M. Munih, "Rotation and orientation," in Introduction to Robotics. Springer, 2013, pp. 9-36. 